\title{
UNIQUENESS RESULTS FOR ELLIPTIC PROBLEMS WITH SINGULAR DATA
}

\author{
LOREDANA CASO
}

Received 22 December 2005; Revised 22 May 2006; Accepted 12 June 2006

We obtain some uniqueness results for the Dirichlet problem for second-order elliptic equations in an unbounded open set $\Omega$ without the cone property, and with data depending on appropriate weight functions. The leading coefficients of the elliptic operator are VMO functions. The hypotheses on the other coefficients involve the weight function.

Copyright (c) 2006 Loredana Caso. This is an open access article distributed under the Creative Commons Attribution License, which permits unrestricted use, distribution, and reproduction in any medium, provided the original work is properly cited.

\section{Introduction}

Let $\Omega$ be an open subset of $\mathbb{R}^{n}, n \geq 3$. Consider in $\Omega$ the uniformly elliptic differential operator with measurable coefficients

$$
L=-\sum_{i, j=1}^{n} a_{i j} \frac{\partial^{2}}{\partial x_{i} \partial x_{j}}+\sum_{i=1}^{n} a_{i} \frac{\partial}{\partial x_{i}}+a
$$

and the Dirichlet problem

$$
L u=0, \quad u \in W^{2, p}(\Omega) \cap \stackrel{o}{W^{1, p}}(\Omega)
$$

with $p \in] 1,+\infty[$.

Suppose that $\Omega$ verifies suitable regularity assumptions.

If $p \geq n, a_{i j} \in L^{\infty}(\Omega)(i, j=1, \ldots, n)$, and the coefficients $a_{i}(i=1, \ldots, n), a$ satisfy certain local summability conditions (with $a>0$ ), then it is possible to obtain a uniqueness result for the problem (D) using a classical result of Alexandrov and Pucci (see [17] for the case of bounded open sets and [6, Section 1] for the unbounded case).

If $p<n$, some more assumptions on the $a_{i j}$ 's are necessary to get uniqueness results for the problem (D). If $\Omega$ is bounded, problem (D) has been widely studied by several authors under various hypotheses on the leading coefficients. In particular, if the coefficients $a_{i j}$ 
belong to the space $C^{o}(\bar{\Omega})$, then uniqueness results for problem (D) have been obtained (see [12-15]). On the other hand, when the coefficients $a_{i j}$ are required to be discontinuous, the classical result by Miranda [16] must be quoted, where the author assumed that the $a_{i j}$ 's belong to $W^{1, n}(\Omega)$ (and consider the case $p=2$ ). More recently, a relevant contribution has been given in $[11,22]$, where the coefficients $a_{i j}$ are supposed to be in the class VMO and $p \in] 1, \infty\left[\right.$; observe here that VMO contains both classes $C^{o}(\bar{\Omega})$ and $W^{1, n}(\Omega)$ (see [10]). If $\Omega$ is unbounded, uniqueness results for problem (D), under assumptions similar to those required in [16], have been for istance obtained in $[4,18,19]$ with $p=2$ and in [5] with $p \in] 1, \infty$ [. Moreover, futher uniqueness results for (D), when the $a_{i j}$ 's are in VMO and $\left.p \in\right] 1, \infty[$, can be found in $[6,9]$.

Suppose now that $\Omega$ has singular boundary. In [8], a problem of type (D) has been investigated, with $\left(a_{i j}\right)_{x_{k}}, a_{i}$ and $a$ singular near a nonempty subset $S_{\rho}$ of $\partial \Omega$, and $p=2$. In particular, the data are supposed to be depending on an appropriate weight function $\rho$ related to the distance function from $S_{\rho}$.

The aim of this paper is to obtain uniqueness results for a Dirichlet problem of type (D) under hypotheses weaker than those of [8] on the $a_{i j}$ 's, and with $p>1$. More precisely, if there exist extensions $a_{i j}^{o}$ of the coefficients $a_{i j}(i, j=1, \ldots, n)$ in $\operatorname{VMO}\left(\Omega_{o}\right) \cap L^{\infty}\left(\Omega_{o}\right)$, where $\Omega_{o}$ is a regular open set containing $\Omega$, and the functions $\rho a_{i}(i=1, \ldots, n), \rho^{2} a$ are assumed to be bounded with $\operatorname{essinf}_{\Omega} \rho^{2} a>0$, we can prove a uniqueness result for the problem

$$
L u=0, \quad u \in W_{\text {loc }}^{2, p}\left(\bar{\Omega} \backslash S_{\rho}\right) \cap \stackrel{o}{W}_{\text {loc }}^{1, p}\left(\bar{\Omega} \backslash S_{\rho}\right) \cap L_{t}^{p}(\Omega),
$$

where $L_{t}^{p}(\Omega), t \in \mathbb{R}$, is a weighted Sobolev space.

Observe that if $S_{\rho}=\partial \Omega$ and $\Omega$ has the segment property, we are able to deduce from the above result that the problem

$$
u \in W_{\text {loc }}^{2, p}(\Omega) \cap L^{p}(\Omega), \quad L u=0,
$$

admits only the trivial solution.

\section{Notation and function spaces}

Let $G$ be any Lebesgue measurable subset of $\mathbb{R}^{n}$ and let $\Sigma(G)$ be the collection of all Lebesgue measurable subsets of $G$. If $F \in \Sigma(G)$, denote by $|F|$ the Lebesgue measure of $F$ and by $\mathscr{D}(F)$ the class of restrictions to $F$ of functions $\zeta \in C_{o}^{\infty}\left(\mathbb{R}^{n}\right)$ with $\bar{F} \cap \operatorname{supp} \zeta \subseteq F$. Moreover, for $p \in[1,+\infty]$, let $L_{\text {loc }}^{p}(F)$ be the class of functions $g$ such that $\zeta g \in L^{p}(F)$ for all $\zeta \in \mathscr{D}(F)$.

Let $\Omega$ be an open subset of $\mathbb{R}^{n}$. We put

$$
\Omega(x, r)=\Omega \cap B(x, r) \quad \forall x \in \mathbb{R}^{n}, \forall r \in \mathbb{R}_{+},
$$

where $B(x, r)$ is the open ball of radius $r$ centered at $x$.

Denote by $\mathscr{A}(\Omega)$ the class of all measurable functions $\rho: \Omega \rightarrow \mathbb{R}_{+}$such that

$$
\gamma^{-1} \rho(y) \leq \rho(x) \leq \gamma \rho(y) \quad \forall y \in \Omega, \forall x \in \Omega(y, \rho(y)),
$$


where $y \in \mathbb{R}_{+}$is independent of $x$ and $y$. For $\rho \in \mathscr{A}(\Omega)$, we put

$$
S_{\rho}=\left\{z \in \partial \Omega: \lim _{x \rightarrow z} \rho(x)=0\right\} .
$$

It is known that

$$
\rho \in L_{\mathrm{loc}}^{\infty}(\bar{\Omega}), \quad \rho^{-1} \in L_{\mathrm{loc}}^{\infty}\left(\bar{\Omega} \backslash S_{\rho}\right)
$$

and, if $S_{\rho} \neq \varnothing$,

$$
\rho(x) \leq \operatorname{dist}\left(x, S_{\rho}\right) \quad \forall x \in \Omega
$$

(see $[7,20])$.

If $r \in \mathbb{N}, 1 \leq p \leq+\infty, s \in \mathbb{R}$, and $\rho \in \mathscr{A}(\Omega)$, we consider the space $W_{s}^{r, p}(\Omega)$ of distributions $u$ on $\Omega$ such that $\rho^{s+|\alpha|-r} \partial^{\alpha} u \in L^{p}(\Omega)$ for $|\alpha| \leq r$, equipped with the norm

$$
\|u\|_{W_{s}^{r, p}(\Omega)}=\sum_{|\alpha| \leq r}\left\|\rho^{s+|\alpha|-r} \partial^{\alpha} u\right\|_{L^{p}(\Omega)}
$$

Moreover, we denote by $\stackrel{o}{W}_{s}^{r, p}(\Omega)$ the closure of $C_{o}^{\infty}(\Omega)$ in $W_{s}^{r, p}(\Omega)$ and put $W_{s}^{0, p}(\Omega)=$ $L_{s}^{p}(\Omega)$. A detailed account of properties of the above-defined function spaces can be found in [21].

If $\Omega$ has the property

$$
\left.\left.|\Omega(x, r)| \geq A r^{n} \quad \forall x \in \Omega, \forall r \in\right] 0,1\right]
$$

where $A$ is a positive constant independent of $x$ and $r$, it is possible to consider the space $\operatorname{BMO}(\Omega, t)\left(t \in \mathbb{R}_{+}\right)$of functions $g \in L_{\text {loc }}^{1}(\bar{\Omega})$ such that

$$
[g]_{\mathrm{BMO}(\Omega, t)}=\sup _{\substack{x \in \Omega \\ r \in] 0, t]}} f_{\Omega(x, r)}\left|g-f_{\Omega(x, r)} g\right| d y<+\infty,
$$

where $f_{\Omega(x, r)} g d y=(1 /|\Omega(x, r)|) \int_{\Omega(x, r)} g d y$. We will say that $g \in \operatorname{VMO}(\Omega)$ if $g \in \operatorname{BMO}(\Omega)=$ $\operatorname{BMO}\left(\Omega, t_{A}\right)$, where

$$
t_{A}=\sup _{t \in \mathbb{R}_{+}}\left(\sup _{\substack{x \in \Omega \\ r \in] 0, t]}} \frac{r^{n}}{|\Omega(x, r)|} \leq \frac{1}{A}\right)
$$

and $[g]_{\mathrm{BMO}(\Omega, t)} \rightarrow 0$ for $t \rightarrow 0^{+}$. 
4 Uniqueness results for elliptic problems with singular data

\section{Some density results}

Let $\rho \in \mathscr{A}(\Omega)$. We consider the following conditions on $\rho$.

$\left(i_{1}\right)$ There exists an open subset $\Omega_{o}$ of $\mathbb{R}^{n}$ with the segment property such that

$$
\Omega \subset \Omega_{o}, \quad \partial \Omega \backslash S_{\rho} \subset \partial \Omega_{o}
$$

(i i $_{2} H=\inf _{\Omega} \rho^{-n}(x)|\Omega(x, \rho(x))| \in \mathbb{R}_{+}$.

Remark 3.1. If condition $\left(\mathrm{i}_{2}\right)$ holds, then it is possible to find a function $\sigma \in \mathscr{A}(\Omega) \cap$ $C^{\infty}(\Omega) \cap C^{0,1}(\bar{\Omega})$ which is equivalent to $\rho$ and such that

$$
\left|\partial^{\alpha} \sigma(x)\right| \leq c_{\alpha} \sigma^{1-|\alpha|}(x) \quad \forall x \in \Omega, \forall \alpha \in \mathbb{N}_{o}^{n},
$$

where $c_{\alpha}$ is independent of $x$ (see [20]).

Fix $r \in \mathbb{N}$ and $p \in\left[1,+\infty\left[\right.\right.$. We denote by $\stackrel{o}{W^{r}, p}\left(\bar{\Omega} \backslash S_{\rho}\right)$ the space of distributions $u$ on $\Omega$ such that

$$
u \in W^{r, p}(\Omega), \quad \operatorname{supp} u \subset \bar{\Omega} \backslash S_{\rho}
$$

Lemma 3.2. Assume that condition $\left(i_{1}\right)$ holds. Then $\mathscr{D}\left(\bar{\Omega} \backslash S_{\rho}\right)$ is dense in $\stackrel{o}{W^{r}, p}\left(\bar{\Omega} \backslash S_{\rho}\right)$.

Proof. Fix $u \in \stackrel{o}{W^{r}, p}\left(\bar{\Omega} \backslash S_{\rho}\right)$ and denote by $u_{o}$ the zero extension of $u$ to $\Omega_{o}$. It is easy to prove that $u_{o}$ belongs to $W^{r, p}\left(\Omega_{o}\right)$. It follows from $\left(i_{1}\right)$ that there exists a sequence $\left\{u_{k}\right\}_{k \in \mathbb{N}} \subset \mathscr{D}\left(\bar{\Omega}_{o}\right)$ such that

$$
u_{k} \longrightarrow u_{o} \quad \text { in } W^{r, p}\left(\Omega_{o}\right)
$$

(see [1, Theorem 3.18]).

Let $\psi \in \mathscr{D}\left(\bar{\Omega} \backslash S_{\rho}\right)$ such that $\psi=1$ on supp $u$. Observe that $\left\{\psi u_{k}\right\}_{k \in \mathbb{N}} \subset \mathscr{D}\left(\bar{\Omega} \backslash S_{\rho}\right)$ and

$$
\left\|\psi u_{k}-u\right\|_{W^{r, p}(\Omega)} \leq\left\|\psi\left(u_{k}-u_{o}\right)\right\|_{W^{r, p}\left(\Omega_{o}\right)} \leq c_{1}\left\|u_{k}-u_{o}\right\|_{W^{r, p}\left(\Omega_{o}\right)},
$$

where $c_{1}$ depends on $n, \psi$. Thus the statement is a consequence of (3.4).

Lemma 3.3. Assume that conditions $\left(i_{1}\right)$ and $\left(i_{2}\right)$ hold. Then $\mathscr{D}\left(\bar{\Omega} \backslash S_{\rho}\right)$ is dense in $W_{s}^{r, p}(\Omega)$. Proof. It follows from $\left(\mathrm{i}_{1}\right),\left(\mathrm{i}_{2}\right)$, and [20, Theorem 4.1] that there exists a sequence $\left\{\delta_{k}\right\}_{k \in \mathbb{N}}$ $\subset \mathscr{D}\left(\bar{\Omega} \backslash S_{\rho}\right)$ such that

$$
\begin{gathered}
\lim _{k \rightarrow+\infty} \partial^{\alpha}\left(1-\delta_{k}(x)\right)=0 \quad \forall x \in \Omega, \forall \alpha \in \mathbb{N}_{o}^{n}, \\
\sup _{k \in \mathbb{N}}\left|\partial^{\alpha} \delta_{k}(x)\right| \leq c_{\alpha} \rho^{-|\alpha|}(x) \quad \forall x \in \Omega, \forall \alpha \in \mathbb{N}_{o}^{n},
\end{gathered}
$$

where $c_{\alpha}$ is independent of $x$.

Fix $u \in W_{s}^{r, p}(\Omega)$. Observe that condition (3.7) implies that $\delta_{k} u \in W_{s}^{r, p}(\Omega)$ for all $k \in$ N. Moreover, by (3.6) we have that

$$
\delta_{k} u \longrightarrow u \text { in } W_{s}^{r, p}(\Omega) .
$$


On the other hand, using (2.4), it is easy to show that $\delta_{k} u \in W^{r, p}(\Omega)$, and so $\delta_{k} u \in$ $W^{r, p}\left(\bar{\Omega} \backslash S_{\rho}\right)$. For each $k \in \mathbb{N}$, Lemma 3.2 yields that there exists a sequence $\left\{u_{h}^{k}\right\}_{h \in \mathbb{N}} \subset$ $\mathscr{D}\left(\bar{\Omega} \backslash S_{\rho}\right)$ such that

$$
u_{h}^{k} \longrightarrow \delta_{k} u \quad \text { in } W^{r, p}(\Omega)
$$

Moreover, let $\psi_{k} \in C_{o}^{\infty}\left(\mathbb{R}^{n}\right)$ such that $\psi_{k}=1$ on $\operatorname{supp}\left(\delta_{k} u\right)$. Thus by (2.4), we have

$$
\left\|\psi_{k} u_{h}^{k}-\delta_{k} u\right\|_{W_{s}^{r, p}(\Omega)} \leq c_{1}\left\|u_{h}^{k}-\delta_{k} u\right\|_{W^{r, p}(\Omega)},
$$

where $c_{1} \in \mathbb{R}_{+}$depends on $\rho, r, s, k$. It follows from (3.9) that there exists $h_{k} \in \mathbb{N}$ such that

$$
\left\|\psi_{k} u_{h_{k}}^{k}-\delta_{k} u\right\|_{W_{s}^{r, p}(\Omega)} \leq \frac{1}{k}
$$

If $\varphi_{k}=\psi_{k} u_{h_{k}}^{k}, k \in \mathbb{N}$, we obtain from (3.8) and (3.11) that

$$
\varphi_{k} \longrightarrow u \quad \text { in } W_{s}^{r, p}(\Omega)
$$

and the lemma is proved.

If $r \in \mathbb{N}, 1 \leq \underset{o}{p}<+\infty$, we will denote by $\stackrel{o}{W}_{\text {loc }}^{r, p}\left(\bar{\Omega} \backslash S_{\rho}\right)$ the set of distributions $u$ on $\Omega$ such that $\zeta u \in \stackrel{o}{W^{r}, p}(\Omega)$ for any $\zeta \in \mathscr{D}\left(\bar{\Omega} \backslash S_{\rho}\right)$.

Lemma 3.4. Assume that conditions $\left(i_{1}\right)$ and $\left(i_{2}\right)$ hold. Then

$$
\stackrel{o}{W}{ }_{\text {loc }}^{r, p}\left(\bar{\Omega} \backslash S_{\rho}\right) \cap W_{s}^{r, p}(\Omega)=\stackrel{o}{W}_{s}^{r, p}(\Omega) .
$$

Proof. It is clearly enough to show that

$$
\stackrel{o}{W}{ }_{\text {loc }}^{r, p}\left(\bar{\Omega} \backslash S_{\rho}\right) \cap W_{s}^{r, p}(\Omega) \subseteq \stackrel{o}{W}{ }_{s}^{r, p}(\Omega) .
$$

Let $u \in \stackrel{o}{W}_{\text {loc }}^{r, p}\left(\bar{\Omega} \backslash S_{\rho}\right) \cap W_{s}^{r, p}(\Omega)$ and consider a sequence $\left\{\delta_{k}\right\}_{k \in \mathbb{N}} \subset \mathscr{D}\left(\bar{\Omega} \backslash S_{\rho}\right)$ satisfying (3.6) and (3.7). Since each $\delta_{k} u$ belongs to $W^{r, p}(\Omega)$, for any $k \in \mathbb{N}$, there exists a sequence $\left\{u_{h}^{k}\right\}_{h \in \mathbb{N}} \subset C_{o}^{\infty}(\Omega)$ such that

$$
u_{h}^{k} \longrightarrow \delta_{k} u \quad \text { in } W^{r, p}(\Omega)
$$

Let $\psi_{k} \in C_{o}^{\infty}\left(\mathbb{R}^{n}\right)$ such that $\psi_{k}=1$ on $\operatorname{supp}\left(\delta_{k} u\right)$. Since $\psi_{k} u_{h}^{k} \in C_{o}^{\infty}(\Omega)$, the same argument used in Lemma 3.3 allows to deduce from (3.15) that for every $k \in \mathbb{N}$, there exists $h_{k} \in \mathbb{N}$ such that

$$
\left\|\psi_{k} u_{h_{k}}^{k}-\delta_{k} u\right\|_{W_{s}^{r, p}(\Omega)} \leq \frac{1}{k}
$$

We put $\varphi_{k}=\psi_{k} u_{h_{k}}^{k}$ for each $k$. Therefore it follows from (3.16) that

$$
\left\|\varphi_{k}-u\right\|_{W_{s}^{r, p}(\Omega)} \leq \frac{1}{k}+\left\|\delta_{k} u-u\right\|_{W_{s}^{r, p}(\Omega)} .
$$


6 Uniqueness results for elliptic problems with singular data

As the sequence $\left\{\delta_{k}\right\}_{k \in \mathbb{N}}$ satisfies (3.8), (3.17) yields that the sequence $\left\{\varphi_{k}\right\}_{k \in \mathbb{N}}$ converges to $u$ in $W_{s}^{r, p}(\Omega)$, and hence (3.14) holds.

\section{Main results}

Let $\Omega$ be an open subset of $\mathbb{R}^{n}, n \geq 3$, with the segment property. Fix $\rho \in \mathscr{A}(\Omega) \cap L^{\infty}(\Omega)$ and consider the following condition on $\Omega$.

$\left(h_{1}\right)$ There exists an open subset $\Omega_{o}$ of $\mathbb{R}^{n}$ with the uniform $C^{1,1}$-regularity property, such that

$$
\Omega \subset \Omega_{o}, \quad \partial \Omega \backslash S_{\rho} \subset \partial \Omega_{o}
$$

Remark 4.1. If condition $\left(\mathrm{h}_{1}\right)$ holds and $\rho \in \mathscr{A}(\Omega) \cap L^{\infty}(\Omega)$, then $\Omega$ satisfies ( $\mathrm{i}_{2}$ ) (see [20]).

Let $p \in] 1,+\infty[$, and let $L$ be the differential operator in $\Omega$ defined by

$$
L=-\sum_{i, j=1}^{n} a_{i j} \frac{\partial^{2}}{\partial x_{i} \partial x_{j}}+\sum_{i=1}^{n} a_{i} \frac{\partial}{\partial x_{i}}+a
$$

Consider the following conditions on the coefficients of $L$ :

$\left(\mathrm{h}_{2}\right)$ there exist extensions $a_{i j}^{o}$ of $a_{i j}$ to $\Omega_{o}$ such that

$$
\begin{gathered}
a_{i j}^{o}=a_{j i}^{o} \in L^{\infty}\left(\Omega_{o}\right) \cap \operatorname{VMO}\left(\Omega_{o}\right), \quad i, j=1, \ldots, n, \\
\exists v \in \mathbb{R}_{+}: \sum_{i, j=1}^{n} a_{i j}^{o} \xi_{i} \xi_{j} \geq v|\xi|^{2} \quad \text { a.e. in } \Omega_{o}, \forall \xi \in \mathbb{R}^{n},
\end{gathered}
$$

$\left(\mathrm{h}_{3}\right)$

$$
\begin{gathered}
a_{i} \in L_{1}^{\infty}(\Omega), \quad i=1, \ldots, n, a \in L_{2}^{\infty}(\Omega), \\
a_{o}=\underset{\Omega}{\operatorname{essinf}}\left(\sigma^{2}(x) a(x)\right)>0,
\end{gathered}
$$

where $\sigma$ is the function defined in Remark 3.1.

Moreover, we suppose that the following hypothesis on $\rho$ holds:

$\left(\mathrm{h}_{4}\right)$

$$
\lim _{k \rightarrow+\infty}\left(\sup _{\Omega \backslash \Omega_{k}}\left((\sigma(x))_{x}+\sigma(x)(\sigma(x))_{x x}\right)\right)=0
$$

where

$$
\Omega_{k}=\left\{x \in \Omega: \sigma(x)>\frac{1}{k}\right\}, \quad k \in \mathbb{N}
$$


In the proof of our main theorem, we need the following uniqueness result.

Lemma 4.2. Assume that conditions $\left(h_{1}\right)-\left(h_{4}\right)$ hold and also that $p>n / 2$. Then the problem

$$
\begin{gathered}
L u=0, \quad u \in W_{\text {loc }}^{2, p}(\Omega), \\
\lim _{x \rightarrow x_{o}}\left(\sigma^{s} u\right)(x)=0, \quad \forall x_{o} \in \partial \Omega, \\
\lim _{|x| \rightarrow+\infty}\left(\sigma^{s} u\right)(x)=0, \quad \text { if } \Omega \text { is unbounded, }
\end{gathered}
$$

admits only the zero solution.

Proof. The statement can be proved as [2, Corollary 5.4]. In fact, the proof of that result also works if the condition $S_{\rho}=\partial \Omega$ is replaced by the assumption $\left(\mathrm{h}_{1}\right)$.

Theorem 4.3. Suppose that conditions $\left(h_{1}\right)-\left(h_{4}\right)$ are satisfied. Then for any $t \in \mathbb{R}$, the problem

$$
u \in W_{\text {loc }}^{2, p}\left(\bar{\Omega} \backslash S_{\rho}\right) \cap \stackrel{o}{W_{\text {loc }}^{1, p}}\left(\bar{\Omega} \backslash S_{\rho}\right) \cap L_{t}^{p}(\Omega), \quad L u=0,
$$

admits only the zero solution.

Proof. Let $u$ be a solution of the problem (4.8). It follows from [3, Theorem 5.2] that $u \in W_{t+2}^{2, p}(\Omega)$. Moreover, $u$ belongs to $W_{t+1}^{1, p}(\Omega)$, and hence Lemma 3.4 yields that $u \in$ $W_{t+2}^{2, p}(\Omega) \cap W_{t+1}^{1, p}(\Omega)$. Using Remark 3.1, it is easy to prove that

$$
\sigma^{t+2} u \in W^{2, p}(\Omega) \cap \stackrel{o}{W}^{1, p}(\Omega) .
$$

Put $v=\sigma^{t+2} u$ and denote by $v_{o}$ the zero extension of $v$ to $\Omega_{o}$. Then

$$
v_{o} \in W^{2, p}\left(\Omega_{o}\right) \cap \stackrel{o}{W^{1, p}}\left(\Omega_{o}\right)
$$

by Lemma 3.3. Suppose first that $p>n / 2$. By the Sobolev embedding theorem, $v_{o}$ belongs to $C^{0}\left(\bar{\Omega}_{o}\right) \cap W^{1, p}\left(\Omega_{o}\right)$, and hence $\left.v_{o}\right|_{\partial \Omega_{o}}=0$. On the other hand, $v_{o} \in W^{2, p}\left(\Omega_{o}\right)$, so that another application of the Sobolev embedding theorem gives that $\lim _{|x| \rightarrow+\infty} v_{o}(x)=0$. Thus by $\left(\mathrm{h}_{1}\right)$, we have that

$$
\lim _{|x| \rightarrow+\infty}\left(\sigma^{t+2} u\right)(x)=0, \quad\left(\sigma^{t+2} u\right)(x)_{\mid \partial \Omega}=0 .
$$

In this case the statement follows now from Lemma 4.2.

Assume now that $p \in] 1, n / 2]$. Then by the Sobolev embedding theorem, we have that $v_{o} \in L^{q}\left(\Omega_{o}\right)$, where $1 / q \geq 1 / p-2 / n$. It follows from [3, Theorem 5.2] that $v_{o} \in W_{2}^{2, q}\left(\Omega_{o}\right)$, and hence $v_{o}$ belongs to $W^{2, q}\left(\Omega_{o}\right)$ by (2.4). If $q>n / 2$, the previous case can be used to complete the proof. If finally $q \leq n / 2$, an iterated application of [3, Theorem 5.2] yields that $v_{o} \in W^{2, q^{\prime}}\left(\Omega_{o}\right)$ with $q^{\prime}>n / 2$. Thus the first case applies again to complete the proof.

As an application of Theorem 4.3, we consider the case $S_{\rho}=\partial \Omega$ (examples of such situation can for instance be found in [20]). The condition $\left(\mathrm{h}_{1}\right)$ is obviously satisfied by 
each $\Omega_{o} \supset \Omega$ with the uniform $C^{1,1}$-regularity property; in this case, condition $\left(\mathrm{h}_{2}\right)$ means that the coefficients $a_{i j}$ admit extensions outside $\Omega$ in the class $L^{\infty}\left(\Omega_{o}\right) \cap \operatorname{VMO}\left(\Omega_{o}\right)$.

Corollary 4.4. Assume that $\left(h_{2}\right),\left(h_{3}\right),\left(h_{4}\right)$ hold and that $S_{\rho}=\partial \Omega$. Then the problem

$$
u \in W_{\text {loc }}^{2, p}(\Omega) \cap L^{p}(\Omega), \quad L u=0
$$

admits only the zero solution.

Proof. The statement follows from Theorem 4.3 observing that, in this case, $u$ belongs to $\stackrel{o}{W}{ }_{\text {loc }}^{1, p}(\Omega)$.

\section{References}

[1] R. A. Adams, Sobolev Spaces, Academic Press, New York, 1975.

[2] L. Caso, Bounds for elliptic operators in weighted spaces, Journal of Inequalities and Applications 2006 (2006), Article ID 76215, 14 pages.

[3] _ Regularity results for singular elliptic problems, Journal of Function Spaces and Applications 4 (2006), Article ID J171, 17 pages.

[4] L. Caso, P. Cavaliere, and M. Transirico, Existence results for elliptic equations, Journal of Mathematical Analysis and Applications 274 (2002), no. 2, 554-563.

[5] Solvability of the Dirichlet problem in $W^{2, p}$ for elliptic equations with discontinuous coefficients in unbounded domains, Le Matematiche (Catania) 57 (2002), no. 2, 287-302 (2005).

[6] __ Uniqueness results for elliptic equations VMO-coefficients, International Journal of Pure and Applied Mathematics 13 (2004), no. 4, 499-512.

[7] L. Caso and M. Transirico, Some remarks on a class of weight functions, Commentationes Mathematicae Universitatis Carolinae 37 (1996), no. 3, 469-477.

[8] _ The Dirichlet problem for second order elliptic equations with singular data, Acta Mathematica Hungarica 76 (1997), no. 1-2, 1-16.

[9] P. Cavaliere, M. Transirico, and M. Troisi, Uniqueness result for elliptic equations in unbounded domains, Le Matematiche (Catania) 54 (1999), no. 1, 139-146 (2000).

[10] F. Chiarenza, M. Frasca, and P. Longo, Interior $W^{2, p}$ estimates for nondivergence elliptic equations with discontinuous coefficients, Ricerche di Matematica 40 (1991), no. 1, 149-168.

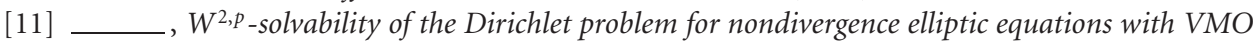
coefficients, Transactions of the American Mathematical Society 336 (1993), no. 2, 841-853.

[12] M. Chicco, Solvability of the Dirichlet problem in $H^{2, p}(\Omega)$ for a class of linear second order elliptic partial differential equations, Bollettino della Unione Matematica Italiana 4 (1971), 374-387.

[13] D. Gilbarg and N. S. Trudinger, Elliptic Partial Differential Equations of Second Order, 2nd ed., Fundamental Principles of Mathematical Sciences, vol. 224, Springer, Berlin, 1983.

[14] D. Greco, Nuove formole integrali di maggiorazione per le soluzioni di un'equazione lineare di tipo ellittico ed applicazioni alla teoria del potenziale, Ricerche di Matematica 5 (1956), 126-149.

[15] A. I. Košelev, On boundedness of $L_{p}$ of derivatives of solutions of elliptic differential equations, Matematicheskij Sbornik. Novaya Seriya 38(80) (1956), 359-372.

[16] C. Miranda, Sulle equazioni ellittiche del secondo ordine di tipo non variazionale, a coefficienti discontinui, Annali di Matematica Pura ed Applicata. Serie Quarta 63 (1963), no. 1, 353-386.

[17] C. Pucci, Limitazioni per soluzioni di equazioni ellittiche, Annali di Matematica Pura ed Applicata. Serie Quarta 74 (1966), no. 1, 15-30.

[18] M. Transirico and M. Troisi, Second-order nonvariational elliptic equations in unbounded open sets, Annali di Matematica Pura ed Applicata. Serie Quarta 152 (1988), 209-226 (Italian).

[19] _ Further contributions to the study of second-order elliptic equations in unbounded open sets, Bollettino della Unione Matemàtica Italiana. Serie VII. B 4 (1990), no. 3, 679-691 (Italian). 
[20] M. Troisi, On a class of weight functions, Rendiconti della Accademia Nazionale delle Scienze detta dei XL. Memorie di Matematica e Applicazioni. Serie V. Parte I 10 (1986), no. 1, 141-152 (Italian).

[21] _ On a class of weighted Sobolev spaces, Rendiconti della Accademia Nazionale delle Scienze detta dei XL. Memorie di Matematica e Applicazioni. Serie V. Parte I 10 (1986), no. 1, 177-189 (Italian).

[22] C. Vitanza, A new contribution to the $W^{2, p}$ regularity for a class of elliptic second order equations with discontinuous coefficients, Le Matematiche (Catania) 48 (1993), no. 2, 287-296 (1994).

Loredana Caso: Dipartimento di Matematica e Informatica, Facoltà di Scienze Matematiche, Fisiche e Naturali (MM. FF. NN.), Università degli Studi di Salerno, Via Ponte don Melillo, Fisciano 84084, Italy

E-mail address: lorcaso@unisa.it 